\title{
Editorial
}

\section{Potencialidades de la cinética del PSA: PET TC con colina y otros escenarios}

\author{
Fernando Daniel Losada López ${ }^{1}$ \\ ${ }^{1}$ Servicio de Diagnóstico por Imágenes, Instituto Alexander Fleming, \\ Buenos Aires, Argentina
}

Rev Argent Radiol 2020;84:83-84.

El estudio de Bergero y col. ${ }^{1}$, "Relación de las cinéticas del PSA en la detección de la recurrencia del cáncer de próstata post prostatectomía radical con la $18 \mathrm{f}$-colina tomografía por emisión de positrones/tomografía computada [PET TC colina (PETC)]," publicado en el presente número, no está dirigido a evaluar pacientes con valores bajos de PSA $(<1-$ $2 \mathrm{ng} / \mathrm{mL}$ ). Sin embargo, sus resultados son similares a los encontrados en publicaciones previas, reafirmando el valor de la cinética del antígeno prostático específico (PSA por su sigla en inglés) (cPSA) en la recurrencia postquirúrgica del cáncer de próstata $(\mathrm{CaP})$, específicamente en relación al tiempo de duplicación del PSA (PSAdt) y a la velocidad del PSA (PSAv).

Resulta de suma importancia el diagnóstico precoz de la recurrencia, ya que la eficacia de las terapias de salvataje es mayor con valores bajos de PSA, es decir, cuando es más probable que la enfermedad sea confinada o locorregional.

Considerando que la aparición de la tomografía por emisión de positrones/tomografía computada (PET TC) con colina ha cambiado el manejo diagnóstico de los pacientes con CaP, resulta importante definir un momento óptimo para su indicación, evitando resultados negativos y costos innecesarios.

Está bien establecida la indicación de PET TC con colina en pacientes con PSA $>1 \mathrm{ng} / \mathrm{mL}$, pero es discutido su uso en pacientes con valores de PSA inferiores, ya que la tasa de detección cae significativamente. ${ }^{2}$

En 2010, Giovacchini ${ }^{2}$ fue el primero en demostrar la relación de la cPSA como predictor de positividad de la PET TC con colina. En dicho trabajo, el porcentaje de casos positivos con PET TC con colina fue del $81 \%$ en pacientes con un PSAdt $<3$ meses, evidenciando una relación inversa entre la positividad de la PET TC y el PSAdt. ${ }^{3}$ La cPSA tiene relación con la agresividad tumoral en la estadificación o reestadificación del $\mathrm{CaP}^{4,5}$ También se ha demostrado la correlación de los valores de la cPSA con pronóstico pobre y/o rápida progresión del CaP. ${ }^{6-9}$ Un PSAdt corto y un valor alto de PSAv son predictores de enfermedad a distancia. Por el contrario, un PSAdt largo y un valor bajo de PSAv son predictores de enfermedad locorregional.

Debido a la fuerte relación entre la cPSA y la tasa de detección de la PET colina, debemos tener en cuenta el PSAdt y la PSAv en la selección de pacientes, antes de referirlos a la PET TC, para optimizar los resultados, especialmente en pacientes con valores bajos de PSA ( $<1-2 \mathrm{ng} / \mathrm{mL}$ ). Además, permitiría seleccionar pacientes oligometastásicos pasibles de terapias localizadas, permitiendo disminuir o postergar las indicaciones de terapias sistémicas.

Resulta difícil de explicar la falta de uso rutinario del PSAdt y de la PSAv en la práctica clínica habitual, dado el impacto potencial, la facilidad de calcular dichos parámetros y su bajo costo.

Por todo lo evidenciado, es factible considerar nuevas aplicaciones potenciales en otros contextos clínicos, como por ejemplo optimizando el uso de PET con antígeno de membrana específico de próstata (PSMA). Un metaanálisis reciente publicó una tasa de detección favorable en pacientes con un PSAdt $<6$ meses. Sin embargo, son necesarios nuevos estudios que avalen dichos resultados con una población de mayor envergadura. ${ }^{10}$

Mamede $^{11}$ reportó resultados similares del PSAdt en pacientes bajo terapia antiandrogénica con valores bajos de PSA $(<0.5 \mathrm{ng} / \mathrm{mL})$ luego de prostatectomía radical, aunque el uso de la cPSA en pacientes tratados con radioterapia o bajo terapias antiandrogénicas es discutido.

Otro escenario potencial que resulta interesante considerar es el de vigilancia activa y el uso de resonancia multiparamétrica, donde todavía se encuentran en discusión la periodicidad de la resonancia y los criterios de progresión a enfermedad clínicamente significativa para tomar medidas activas. Parecería intuitivamente obvio que la cPSA debería tener algún valor predictivo en este contexto. Obtener mejores predicciones significaría menos biopsias negativas y mayor detección de cáncer significativo. Un trabajo de Cooperberg ${ }^{12}$ muestra resultados en esa dirección, ya que incorporaron parámetros distintos al PSAv y PSAdt pero
Address for correspondence Fernando Daniel Losada López, MD, Servicio de Diagnóstico por Imágenes, Instituto Alexander Fleming, Buenos Aires, Argentina (e-mail: fernandolosada83@gmail. com).
DOI https://doi.org/ 10.1055/s-0040-1715806. ISSN $1852-9992$.
Copyright $\odot$ 2020, Sociedad Argentina de Radiología. Publicado por Thieme Revinter Publicações Ltda., Rio de Janeiro, Brazil. Todos los derechos reservados.
License terms

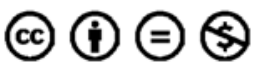


relacionados a la cPSA en un modelo predictivo, potenciando sus resultados. Si continuamos en esta dirección, probablemente encontremos nuevos beneficios de estos parámetros en la práctica clínica. ${ }^{12}$

\section{Bibliografía}

1 Bergero MA, Ríos L, David C, González Piccini CA, Derian E, Fleurquin $\mathrm{M}$, et al. Relación de las cinéticas del PSA en la detección de la recurrencia del cáncer de próstata post prostatectomía radical con la $18 \mathrm{f}$-colina tomografía por emisión de positrones/tomografía computada. [PET TC colina (PETC)]Rev Argent Radiol. 2020;84(03):XX-XX

2 Giovacchini G, Breeuwsma AJ. Restaging prostate cancer patients with biochemical failure with PET/CT and radiolabeled choline. QJ Nucl Med Mol Imaging. 2012;56(04):354-366

3 Pelosi E, Arena V, Skanjeti A, V Pirro, A Douroukas, A Pupi, et al. Role of whole-body $18 \mathrm{~F}$-choline PET/CT in disease detection in patients with biochemical relapse after radical treatment for prostate cancer. Radiol Med. (Torino) 2008;113(06):895-904

4 Vickers AJ, Wolters T, Savage CJ, Cronin AM, O’Brien MF, Pettersson, et al. Prostate-specific antigen velocity for early detection of prostate cancer: result from a large, representative, population-based cohort. Eur Urol. 2009;56(05):753-760

5 Antonarakis ES, Zahurak ML, Lin J, Keizman D, Carducci MA, Eisenberger MA. Changes in PSA kinetics predict metastasisfree survival in men with PSA-recurrent prostate cancer treated with nonhormonal agents: combined analysis of 4 phase II trials. Cancer. 2012;118(06):1533-1542
6 O'Brien MF, Cronin AM, Fearn PA, Smith B, Stasi J, Guillonneau B, et al. Pretreatment prostate-specific antigen (PSA) velocity and doubling time are associated with outcome but neither improves prediction of outcome beyond pretreatment PSA alone in patients treated with radical prostatectomy. J Clin Oncol. 2009;27(22): 3591-3597

7 Schiavina R, Brunocilla E, Borghesi M, Vagnoni V, Castellucci P, Nanni $C$, et al. Diagnostic imaging work-up for disease relapse after radical treatment for prostate cancer: how to differentiate local from systemic disease? The urologist point of view. Rev Esp Med Nucl Imagen Mol. 2013;32(05):310-313

8 Khan MA, Carter HB, Epstein JI, Miller MC, Landis P, Walsh PW, et al. Can prostate specific antigen derivatives and pathological parameters predict significant change in expectant management criteria for prostate cancer? J Urol. 2003;170(6 Pt 1):2274-2278

9 Svatek RS, Shulman M, Choudhary PK, Benaim E. Critical analysis of prostate-specific antigen doubling time calculation methodology. Cancer. 2006;106(05):1047-1053

10 Mestre R, Treglia G, Ferrari M, Pascale M, Mazzara C, Azinwi N, et al. Correlation between PSA kinetics and PSMA-PET in prostate cancer restaging: A meta-analysis. Eur J Clin Invest. 2019;49(03): e13063

11 Mamede M, Ceci F, Castellucci P, Schiavina R, Fuccio C, Nanni C, et al. The role of $11 \mathrm{C}$-choline PET imaging in the early detection of recurrence in surgically treated prostate cancer patients with very low PSA level <0.5 ng/mL. Clin Nucl Med. 2013;38(09):e342-e345

12 Cooperberg M, Brooks J, Faino A, Newcomb L, Kearns J, Carroll P, et al. Refined Analysis of Prostate-specific Antigen Kinetics to Predict Prostate Cancer Active Surveillance Outcomes. Eur Urol. 2018;74(02):211-217 